\title{
Integration Solutions for Video Data in Educational Platforms
}

\author{
http://dx.doi.org/10.3991/ijet.v9i8.3482 \\ A.M. Gabor, R. Vasiu \\ "Politehnica" University of Timisoara, Romania
}

\begin{abstract}
Interdisciplinarity will become more and more not just a way of restructuring content, but also a new method of organizing the teaching process. The need of reusing video data or parts of it in the context or content of diverse courses is often imposed. Successful management of large video data implies using the results obtained on queries based on semantics. This paper aims to present the mode in which video data are modeled in e-Learning platforms trough semantic annotation of the content, using the MPEG-7 standard and specific ontologies of the e-Learning domain and as far as retrieval and reuse of results in specific applications for creating interactive courses.
\end{abstract}

Index Terms - content retrieval, e-Learning, MPEG-7, ontology.

\section{INTRODUCTION}

Currently, special attention to the e-Learning domain is given because of the number and the diversity of the users, from the big companies to the educational institutions. The e-Learning platforms have a unitary structure, specific procedures for administration, documentation, making teaching activities and assuring at the same time interaction between users and groups of users. The e-Learning platform is made of: LMS (Learning Management System), LCMS (Learning Content Management System) and a WEB portal [1]. LMS is a software application for administration, documentation, reviewing, course report, classroom report, events and user report. LCM (Learning Content Management System) is a technology tied to the management system and publishing content, which will be delivered through LMS (Learning Management System). LCMS creates, stores, manages digital content, also it allows creating, importing, managing and searching some parts of the digital content, known as learning object (LO). These objects include media files, evaluating elements, text, graphics or any other object that creates the content of a course. Many times the need to reuse this objects in context and content of diverse courses arises. This is, in fact, the base philosophy of the educational application portability through SCORM standard, implemented by IEEE Learning Technologies Committee [2]. Remote education started new directions of development as more powerful computers appeared and growing bandwidth for communication and video materials [3]. Video materials have a significant pedagogical impact on users because they transmit complex images made from text, sound and image. Many institutions and universities broadcast video materials through educational platforms. These institutions or "Virtual Universities" are described as organization, which offer learning programs or online practicing utilizing diverse media technologies. Finding content based on the semantic content describes the probability that a video clip to be classified as being a relevant or irrelevant clip as the query made by the user. The query and retrieving the information based on semantics is more efficient than based on the keywords, as it models on human thinking and the results are more relevant for users. The task of retrieving video content has the role of searching in a large quantity of video clips for finding information expressed by the user and to describes the complete frame from the indexing and annotation of the video content, using the MPEG-7 standard and the ontologies from the educational domain, these being described in the first part of the work, followed by the retrieval of video Information using the MPQF query framework and grouping the query results of the user are described in the second section of the work. Eventually, we described in our paper some application, which use the descriptions from our work for making interactive courses for the eLearning platforms.

\section{INDEXING AND SEMANTIC ANNOTATION OF THE VIDEO CONTENT BASED ON ONTOLOGIES}

Video materials have become the most popular multimedia data because of the large volume of information they transmit. In this case the main challenge is indexing the video information for facilitating retrieval of the content in an efficient way. This thing necessitates an annotation of the content that can be made manually or automatically through algorithms so that there will be an accurate description of the media content. Searching for a relevant movie parts, according with the semantic content offers the possibility (for the teacher or student) to reutilize a part of the content. With all this, the users are put sometimes, in the impossibility to find a specific area of information in a video stream, and often is necessary to manually search the information. The indexing task of the content is more complicated as the content is richer semantically. On the e-Learning platforms the searching and retrieval of content can grow from the use of ontologies. Thus, there were identified multiple types of ontologies tied to concept, domain, ontologies specific for teaching and learning but also ontologies about the physical structure of objects. (Mahan and Brooks 2003) [4].

\section{A. Creating ontologies in the educational domain}

Utilizing ontologies in the e-Learning domain has grown in the last years. Researches have been materialized through many projects of which we remind "Trial Solutions" [5], the paper of Dragan G., J. Jovanovic \& Vladan D. (2007) [6] and also with applicability in the medical domain, the paper of S. Colantonio et al (2009) [7]. Utilizing Web semantic technologies in the context of e- 
Learning has been applied by Panteleyev (2002) [8], but also through making some intelligent e-Learning spaces in the work of Dolog (2004) [9]. Ontologies based on content (domain), context and structure have been made (Stojanovic, Stoab and Studer 2001) [10] and are compatible with other instruments of navigation and indexing. Previously, ontologies from the e-Learning domain were used only for upgrading metadata of Learning Object (LO). Although such approach is useful because it improves the retrieval of learning objects but does not permit the reutilizing of $\mathrm{LO}$ and incorporate a specific explication of the semantic domain of the LO. A very important part is the use or reuse of the content. This thing implies a representation of the LO structure which will allow the reuse of components. These pieces of content can be upgraded with metadata allowing to search and reuse of the LO in the content and context of the course. Thus there are many types of ontologies for making the LO reusable (fig. 1):

- Metadata ontologies (MO)

- Content structured Ontologies (CSO)

- Domain content Ontologies (DO)

In order to assure an interoperability within the eLearning platform between the content given by the supplier and the produced one there were set some standards necessary for describing the content of the courses and to manage the user profile. Thus, under IEEE Learning Technology Standards Committee (LTSC) IEEE P 1.484.12.1-2002 Learning Object Metadata Working Group, defines the learning object (LO) as being an independent collection of elements with media content based on interactivity, architecture, context and metadata (used for annotation and search). Annotations based on the ontology "semantic annotation" describe the video resource and allow thus, a bond between the structured data and unstructured ones (ontology, text). Ontologies for the educational domain are based on the level of expressivity (or formal) which conditions a certain form of ontology that will be utilized in the automatic reasoning [11]. Some important aspects which need to be considered for creating an e-Learning ontology were described based on [12] the idea of interoperability or information exchange:

- -Learner Profile

- -E-Learning context

- -Interactivity

- -Evaluation/opinion

LOM (Learning Object Metadata) is similar with the Dublin Core metadata standard but is specific to eLearning platforms and describes the content of a course using metadata attributes at a higher level (semantic). It also offers specific e-Learning domain attributes like the difficulty of a course, type of course, lessons that compose the course, length of the course and the structure of the course. The purpose of LOM (Learning Objective Metadata) is to offer support for utilizing or reutilizing of LO (Learning Object) in the educational platforms (fig.2).

It is important to classify the multimedia content based on ontologies to increase the semantic description of the content but also to reduce metadata which sometimes are expensive to make [13]. Learning Object Metadata describes LO (Learning Object) and similar digital resources used for supporting the learning technology. Some standards of metadata were proposed like Isaac`s (2004) [14] as

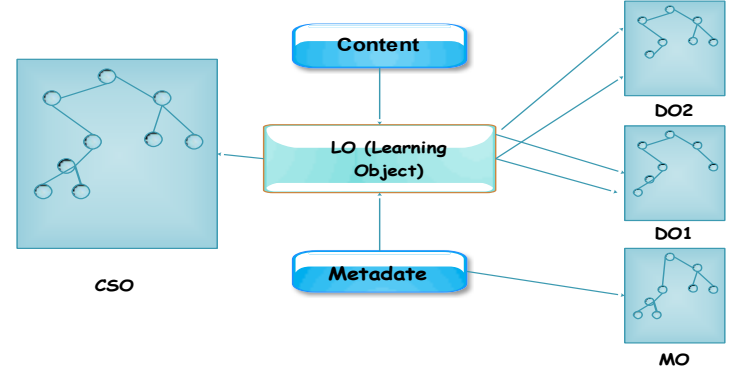

Figure 1. E-Learning Ontology

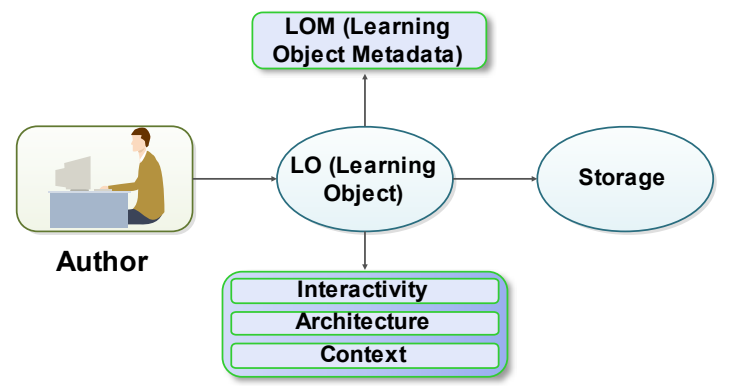

Figure 2. The Learning Object

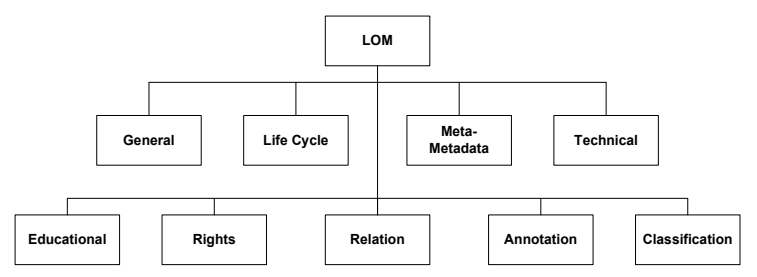

Figure 3. LOM Elements Hierarchy

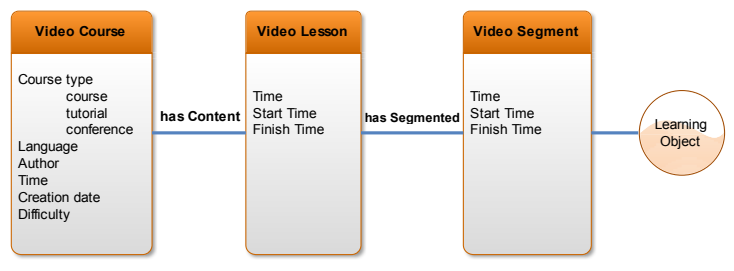

Figure 4. Content Ontology Description

Dublin Core [15], LOM [16], Vra Coreh [17] which are directly tied to the media content presentation on a certain domain and offer standardized schemes of descriptions and a well-defined syntax. SCROM (Sharable Content Object Reference Model) uses the XML language to define Course Structure Format which represents the structure of the courses so that educational video materials to interoperate with other platforms. Structural ontology content refers to an ontological representation designed to describe LOM with the help of metadata.

According to IEEE Learning Technology Standards Committee (LTSC), IEEE P1848.12.1-2002 Learning Object Metadata Working Group, learning object (LO) is defined as being anything digital or non-digital that can be used or reused in the e-Learning technology. Also, a LO can be considered to be an independent collection of media content elements (interactivity, architecture, context) and metadata. More LO are put together by authors to form courses and then are delivered for use.

Once the ontology of the learning object (LO) has been created, it can be integrated so it will allow the efficient 
retrieval of content and the possibility to be reused (fig. 4). SCORM reflects on the tendency to unite metadata specifications as a specialized subset which describes RLO (Reusable Learning Object) based on content by RIO (Reusable Information Object) [18].

\section{B. Video annotation using MPEG-7 standard}

For the video documents there are necessary some supplementary annotations comparatively for those based on text where it is often sufficient just to add an annotation for the generic properties (title, author) for searching and finding content. An important step in efficiently manipulating and visual retrieval is represented by the semantic information indexing so that the system will express the entities and relations which define the content [19]. The base idea for indexing the file in the video e-Learning platforms is based on the types of ontology which define the context, the file structure but also ontologies specific for teaching and learning. MPEG-7 designed by Movie Picture Expert Group (MPEG) is one of the standards largely accepted for describing media content. MPEG-7 offers a rich set of instruments for describing the content, managing the content, navigation and access but also interaction with the user. For this purpose, ontologies specific for the MPEG-7 standard have been made as described in the work of Hunter [20] and Tsinaraki [21].

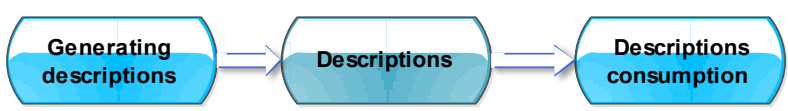

Figure 5. The aim of MPEG-7

MPEG-7 addresses to application from different environments so it offers a flexible framework for describing multimedia data. MPEG-7 does not describe a system for media descriptions, but rather methods and instruments for different content descriptions (fig. 5). The main elements of MPEG-7 are:

- Description tools: descriptors (Ds) which define the syntax and semantic of each element (metadata) and description schemes (DSs) which specify the structure and semantic of the relations between components which can be descriptors but also description schemes.

- Description Definition Language (DDL) which defines the syntax of description tools and allows creating new description schemes.

- System tools to help the binary representation for storing information, synchronizing, management and individual protection.

Therefore, MPEG-7 allows us to create descriptions incorporated in the application extensions using DDL. DDL permits defining of tools for description but also Ds- descriptors and DSs- description schemes, offering means for structuring descriptors (Ds) in the description schemes (DDs). To make the indexing for the video documents for semantic annotation, we used the Polysema application integrating in this, ontological applications created in the e-Learning domain which have been described above. The application permits the display of results coming from the indexing and offers the possibility to merge or delete video clips followed by the annotation of the content, process in which the text will be associated to it. Once the annotation has been made, the application will generate a MPEG-7 file which describes the events and the relations between it, under the form of a file that utilizes the XML language, described in a previous work of ours [22].

\section{MPQF FRAMEWORK FOR RETRIEVING VIDEO CONTENT}

Retrieving video content based on semantic concepts describes the possibility that a video clip to be tied to the concept and classified as relevant or irrelevant. Thus, the task of retrieving the content has the role of searching into a big collection of clips to find the information expressed by the user through query. MP7QF defines the format for query the content and it is responsible for the process of searching and retrieving it. A very important characteristic is in the process of retrieval of data, MP7QF must accept video formats returned by the query formulated by the user (fig. 6).

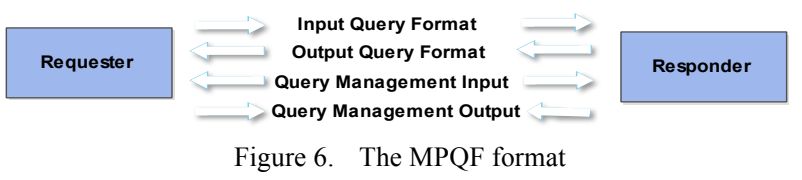

XML has completely changed the way of data exchange between applications because it allows the exchange of heterogeneous information. MPQF is a XML language meaning that all the MPQF instances (query and answer) need XML documents. The integration format of MPQF is Part. 12 of ISO/IEC 15938 and expresses the conditions of metadata to use the privileges offered by XML, RDF and OWL. The objective of the MP7QF framework is to assure an interface for the MPEG-7 database which will allow retrieving of multimedia data by the user [23]. The student enrolled in a certain course can make queries in multiple databases and the results of the query to be based on his preferences and the utilization history. In order to use MP7QF framework on the e-Learning platforms, it must meet the following requirements :

- It must allow searching multiple databases

- Accepting multiple formats returned by the query process of retrieving multimedia data

- The MP7QF must provide support for different queries:

- Description queries, which are based on textual descriptions but using the schemes and depiction of MPEG-7 descriptions.

- Queries based on example where time-space relations will be used for query, but also the semantic and low characteristics

- Queries based on the specifications of the MPEG-7 standard. It will be used for descriptors

- Queries (of color, of texture, etc) and description schemes.

- Queries based on the preferences and history of the user

\section{INTEGRATION OF VIDEO DATA IN MAKING INTERACTIVE SMIL AND FLASH COURSES}

Using of the interactive video courses in the e-Learning platforms is a modern method for accomplishing efficiently learning goals. Interactive e-Learning courses represent a powerful teaching tool in universities and institutions offering many benefits through lowering the cost of implementation, projection, course transport, but also 
through standardization of content for all students. Using centralized materials, control of student formation is gained. An interactive course is composed of more components having associated diverse types of multimedia data (text, audio, video, images) which can be played in real time in a sequential manner, parallel or in a combined way. The MPEG-7 standard offers the advantage of describing the video content through semantic annotation and further to help at searching, navigating and retrieving it in an efficient way for using it in the interactive courses. The mp7 file generated by the application is in fact a XML file which can be used for loading external video data in Flash and SMIL assuring a method of structuring content, easy to understand and accessible to the students. The advantage of utilizing interactive courses in the educational domain is that it will utilize only references to the e-Learning Object, references obtained from semantic indexing of the video content and the resulted $\mathrm{mp} 7$ file has a reduced size.

\section{A. Loading the external XML Data in Flash}

Flash can read and store the XML (Extensible Makeup Language) using ActionScript. The XML language is a popular method of storing external data. This means that Flash is capable of creating dynamic applications based on external data. The mp7 file generated by the Polysema application, is a file based on the XML language, the tags from the file are also known as nodes, so that each node can contain a series of specific optional attributes so as to the format of a node is :

$$
<\text { node-name attribute="value"/> }
$$

XML can be used to load external data in Flash for assuring an easy to use method of structuring content in interactive courses. In the next example, there is described the loading method in Flash, of a document, having a XML structure using ActionScript. Thus, Flash can obtain access to video data in real time for creating e-Learning courses which are more interesting and attractive for students [24].

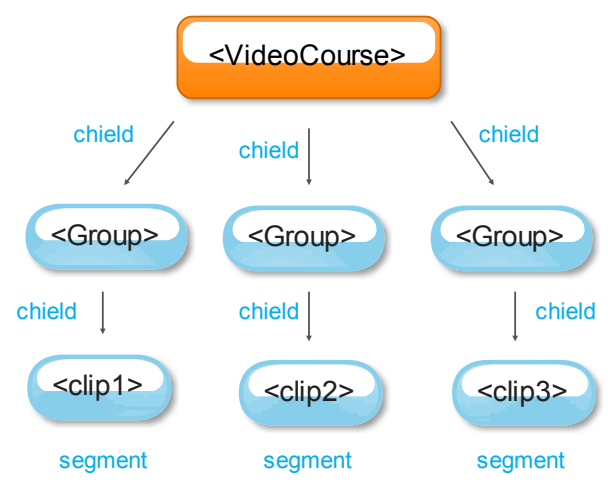

Figure 7. Structure of video courses

// Set the ignoreWhite property to true (default value is false).

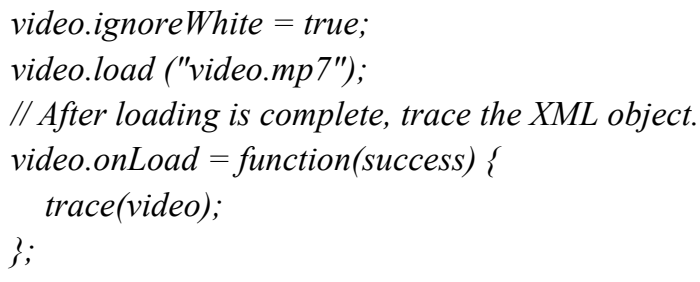

// Load the XML into the video object.

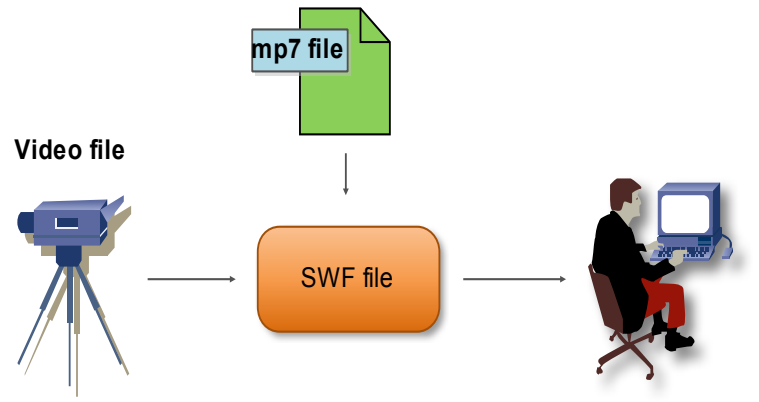

Figure 8. Playing courses

For loading the data the use of URLLoader Class will be needed and during processing the XML Class. This process will be structured in 3 stages :

- Loading the mp7 file using the URLLoader

- Processing using the XML Class

- SWF loads all video thumbnails

\section{B. SMIL Language}

It is a $\mathrm{W} 3 \mathrm{C}$ recommendation and it is a language based on XML, with the purpose of facilitating interactive multimedia presentations. The contents of a synchronous SMIL presentation can be made up from more video streaming real time, into a sequential manner, parallel or combined. The video streaming obtained through query can be utilized in any presentations we want adding (establishing) some temporal and spatial links. Playback of SMIL presentations implies the existence of a specialized player for SMIL documents, examples of such players are RealPlayer, QuickTime, S2M2 etc. SMIL is a language for annotation based on XML for creating hypertext content attractively and without altering the video content. A SMIL file is composed of a succession of elements through which video files are accessed and can be placed in certain regions specified by the user. The position of a clip in a presentation is given either by coordinates or using translation functions.

To specify what will be played in each region defined in the body of the SMIL document, a region attribute will be added on each video object. Thus, the hierarchy of regions and windows defined with the help of the XML syntax in the SMIL presentation specifies rigorously the screen content framing. To localize the video file, the src attribute will specify the file path (fig. 9).

The execution of a video object can be planned to start and stop at precise moments in time in the evolution of the presentation with the attributes "begin" and "end". SMIL is a meta-language indicating information about the next presentation that will be made, tied to the source of the video files, space template and the timeline of the presen-

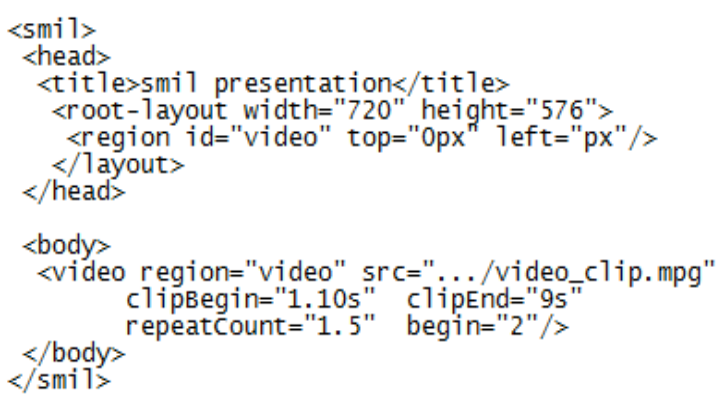

Figure 9. SMIL code 
PAPER

INTEGRATION SOLUTIONS FOR VIDEO DATA IN EDUCATIONAL PLATFORMS

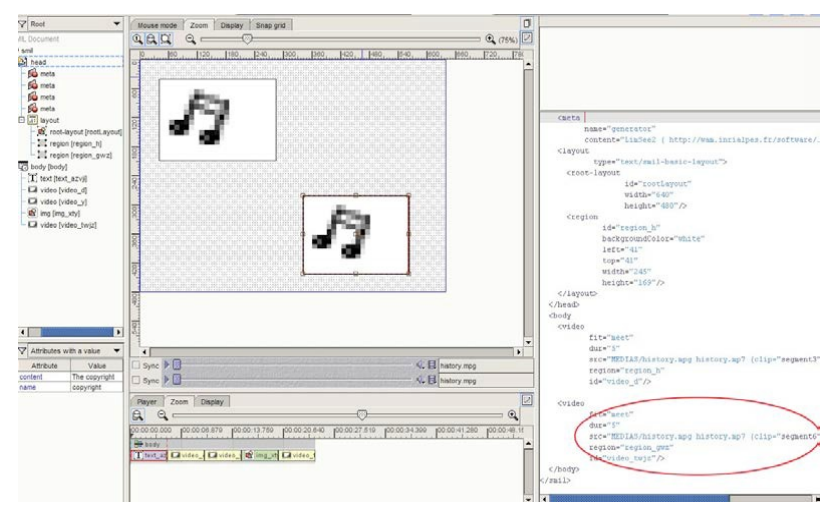

Figure 10. LimSee application

tation. For practical implementation of the above described, we used LimSee application (fig. 10) developed by INRIA [25], which provides the ability to edit spatial and temporal structure of the document providing a tree view of the document structure. We will consider the description associated with video segments to express the MPEG-7 integration within SMIL documents:

$$
<\text { src=video.mpg\#mp7(clip="segment6") }>
$$

Thus, any segment of video stream can be chosen to be integrated in the SMIL document using temporal and spatial description of the mp7 file [26].

\section{CONCLUSIONS AND FUtURE RESEARCH}

The paper aims to present the management of video data in educational platforms combining diverse multimedia technologies from creation to consumption. Thus, I combined annotation technologies based on domain ontology in the e-Learning platforms but also retrieving and reordering techniques of video content. The user can now, manage searching and finding more efficiently thought a large quantity of data necessary information to use or reuse of the video information in the e-Learning courses. Further research will be channeled on creating and experimenting new ways of utilizing video data in educational platforms through creating algorithms that exploit and combine MPEG-7 descriptors, which contain low level information (color, texture, etc.) but also conceptual information about objects and the relation between them.

\section{REFERENCES}

[1] http://www.fluidpower.ro/itfps/Etapa1ITFPS.pdf

[2] IEEE Learning Technology Stardardization Committee Draft Standard for Learning Object Metadata, 18 April 2001

[3] Kathleen Harting \& Margaret J. Erthal „Hystory of Distance Learning", Information Technology, Learning and Performance Journal, vol.23, No.1, mai 2010

[4] Mohan, P., \& Brooks, C. "Learning objects on the Semantic Web" in Procedings of the 3rd IEEE International Conference on Asdvanced Learning Technologies (pp.195-199), Los Alanitos CA : IEEE Compuer Society Press 2003

[5] M.Buffa, S. Dehors, C. Faron-Zucker, P. Sander, "Towards a Corporate Semantic Web Approach in Designing Learning System", In: Proc. of International Workshop on Applications of Semantic Web Technologies for E-Learning, AIED. 2005. p. 73-76.

[6] Gašević, Dragan, Jelena Jovanović, and Vladan Devedžić. "Ontology-based annotation of learning object content." Interactive $\begin{array}{llll}\text { Learning } & \text { Environments } 15.1 & \text { (2007): } & 1-26 \text {. }\end{array}$ http://dx.doi.org/10.1080/10494820600968203

[7] S. Colantonio, O.Salvetti, IB Gurevich \& Y. Trusova „An Ontological Franework for Media Analysis and Mining, Pattern Recog- nition and Image Analysis, vol.19, No.2, 2009 http://dx.doi.org/10.1134/S1054661809020023

[8] Panteleyev M, Puzankov, D., Sazykin, P, Sergeyev, D (2002) "Intelligent educational enviroments based on the Semantic Web technologies". In Proceeding of the IEEE International Conference on Artificial Intelligence Systems, Los Alamitosd, CA: IEEE Computer Society Press, pp.457-462

[9] Dolog, P., Henze, N., Nejdl, W., \& Sintek, M. (2004) "Personalization in distributed e-learning enviroments". In Proceeding of the 13th Inernational Word Wide Web Conference on Altenate Track Paper \& Posters New York, USA Retriered December 4, 2005, pp.170-179 http://dx.doi.org/10.1145/1013367.1013395

[10] Stojanovic, L., Staab, S., \& Studer, R. „eLearning in the Semantic Web" in Proceeding of the word Conference on the WWW and the internet (WebNet 2001) Chesapeake, VA: AACE. pp:.23-27

[11] Madan, D., Pant, A., Kumar, S., Arora, A., "E-learning based on Cloud Computing". International Journal of Research in Computer Science and Software Engineering, 2012, vol. 2.

[12] Casquero O., Portillo J., Ramó, Ovelar R., and Benito M., "iGoogle and gadgets as a platform for integrating institutional and external services," MUPPLE'08, pp. 37-41, 2008

[13] Zhang, L. J., \& Zhou, Q. (2009, July). "CCOA: Cloud computing open architecture. In Web Services", 2009. ICWS 2009. IEEE International Conference on Ieee. pp. 607-616

[14] Isaac, A., and Troncy R., (2004) "Designing and Using an Audiovisual Description Core Ontology". Proceeding of the 14th International Conference on Knowledge Engineering and Knowledge Management, October 2004

[15] Dublin Core Metadata Element Set Version 1.1, http://dublincore.org/documents/dces/

[16] http://tsc.ieee.org/wg12//LOM, IEEE Learning Technology Standards Committee Learning Object Meta-data Working Group, 2002,

[17] http://www.vraweb.org/projects/vracore4/index.html

[18] Aroyo, L., Pokraev, S., \& Brussee, R. (2003). "Preparing SCORM for the semantic web". In On The Move to Meaningful Internet Systems 2003: CoopIS, DOA, and ODBASE (pp. 621-634). Springer Berlin Heidelberg.

[19] Petridis, K., Kompatsiaris, I., Strintzis, M. G., Bloehdorn, S., Handschuh, S., Staab, S. \& Avrithis, Y. S. (2004, November). "Knowledge Representation for Semantic Multimedia Content Analysis and Reasoning". In EWIMT 2004.

[20] Hunter, J (2001) "Adding Multimedia to the Semantic Web Building an MPEG-7 Ontology". The 1st International Semantic Web Working Symposium (SWWS'01) pp 261-281

[21] Tsinaraki, C., Polydoros, P., and Christodoulakis, S. "Interoperability suport for Ontology-based Video Retrieval Applications". Proceeding of 3rd International Conference on Image and Video Retrieval, pp 582-591

[22] Gabor, Andrei Marius, Radu Vasiu. "Interdisciplinarity in eLearning Platforms Based on Textual Annotation." Information and Software Technologies. Springer Berlin Heidelberg, 2012. pp 362-372.

[23] Kerstin Renner. Specification of an MPEG-7 Query Language, University of Pasau, Germany, 2007

[24] Gabor, A. M., R. Vasiu, and L. Gaga. "Video data use in interactive e-Learning courses. A modern method of learning organizing process".ICERI2013 Proceedings (2013) pp: 2184-2190.

[25] http://limsee3.gforge.inria.fr/public-site/

[26] Gabor, A., R. Vasiu. "Smil presentations-use of video data in eLearning Platforms." EDULEARN13 Proceedings (2013) pp: 340347.

\section{AUTHORS}

A. M. Gabor, R. Vasiu are with Department of Communications at "Politehnica" University of Timisoara (marius@cm.upt.ro,radu.vasiu@cm.upt.ro) Romania.

This work has been partially supported by the strategic grant POSDRU 107/1.5/S/77265, inside POSDRU Romania 2007-2013 cofinanced by the European Social Fund - Investing in People. Submitted 12 December 2013. Published as re-submitted by the authors 26 May 2014. 